\title{
Paro Cardiorespiratorio secundario a Hematoma Coronario Intramural en arteria descendente anterior
}

\author{
Cristian Matamala ${ }^{1}$, Carlos Romero², Jorge Sandoval2, Osvaldo Pérez ${ }^{1}$, Alejandro Martínez ${ }^{1}$, Gonzalo Martínez \\ 1 División de Enfermedades Cardiovasculares, Escuela de Medicina, Pontificia Universidad Católica de Chile \\ 2 Clínica Bicentenario, Santiago, Chile
}

\section{Cardiac Arrest associated to Intramural Hematoma of the Anterior Descending Coronary Artery}

A 43 year old woman presented with chest pain followed by cardiac arrest recovered after defibrillation. Coronary angiography revealed a narrowed anterior descending coronary artery but no intraluminal thrombi. Optic coherence tomography showed intramural hematoma and the patient was treated medically with aspirin, clopidogrel and atorvastatin. Two weeks later the pain recurred and coronary angiography revealed similar findings. After medical treatment with heparin followed by aspirin and clopidogrel she has remained stable.

Keywords: Spontaneous coronary artery dissection; coronary intramural hematoma; optic coherence tomography. 
Mujer de 43 años, con antecedente de tabaquismo detenido. Consulta por cuadro brusco de dolor torácico dorsal interescapular, irradiado a región mandibular, de carácter opresivo, asociado a disnea. Una hora luego de iniciado los síntomas sufre paro cardiorespiratorio, recibiendo reanimación cardiopulmonar y desfibrilación con 200J. Una vez estabilizada, fue trasladada a un centro de mayor complejidad. Se obtuvo elctrocardiograma (Figura 1). Exámenes de laboratorio mostraron CK total 1.316 U/L y CKMB 284 U/L, kalemia de $3,5 \mathrm{mEq} / \mathrm{L}$ y leucocitos de 32.270 x 103/uL.

Mediante un AngioTAC de tórax se descartó disección aórtica y TEP. Alrededor de 11 horas luego del paro cardiorespiratorio se realizó coronariografía (Figura 2). La ventriculografía mostró una extensa área de marcada hipoquinesia anterior y antero-apical. Dados estos hallazgos,

Figura 1. Electrocardiograma de 12 derivaciones tomado unas horas después del paro cardíaco. Se observa taquicardia sinusal, con onda $Q$ y $\mathrm{T}$ negativas en pared anterior.

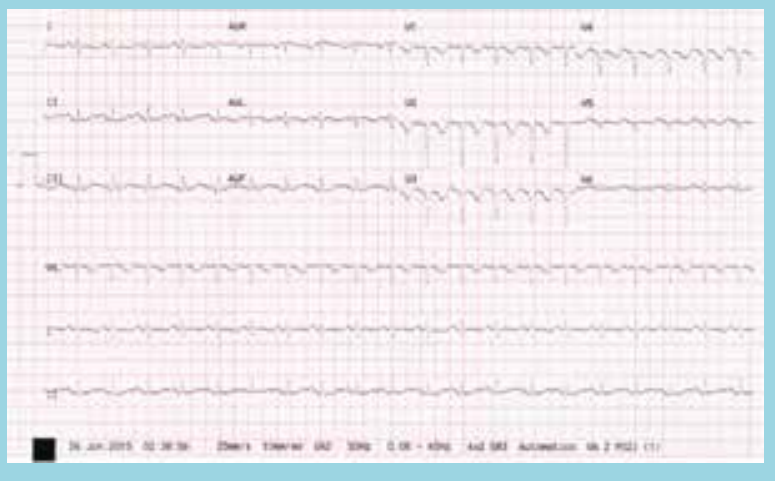

Figura 2. Se observa una estrechez luminal de bordes lisos en los tercios proximal y medio de la arteria Descendente Anterior (ADA). Las flechas se correlacionan con las imágenes intracoronarias obtenida con OCT en la Figura 3

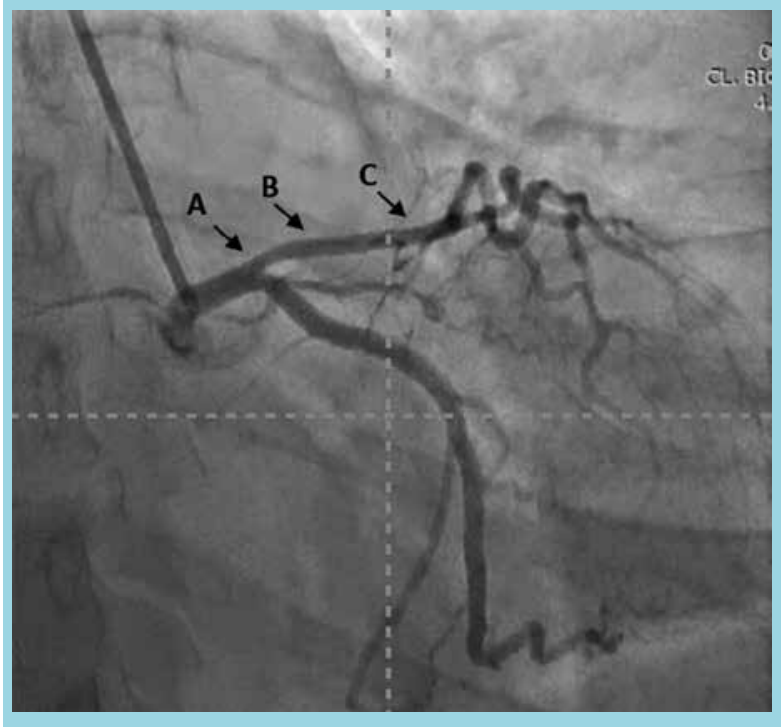

se complementó el estudio con imágenes intracoronarias mediante Tomografía de Coherencia Óptica (OCT). Este estudio demostró claramente la presencia de un hematoma entre las túnicas media y adventicia de la pared vascular (Figura 3).

Dado que el lumen de la arteria se encontraba permeable, se decidió manejar su enfermedad coronaria de forma médica. La paciente fue dada de alta, en buenas condiciones,

Figura 3. OCT muestra extenso hematoma intramural en Arteria Coronaria Descendente Anterior proximal y media, entre la túnica media y la adventicia, que reduce el lumen verdadero. A: segmento más proximal del hematoma, que se extiende hasta el tronco común izquierdo (flecha). En B se aprecia 1: íntima, 2: media y 3: adventicia. En B y C, el asterisco señala el hematoma intramural.
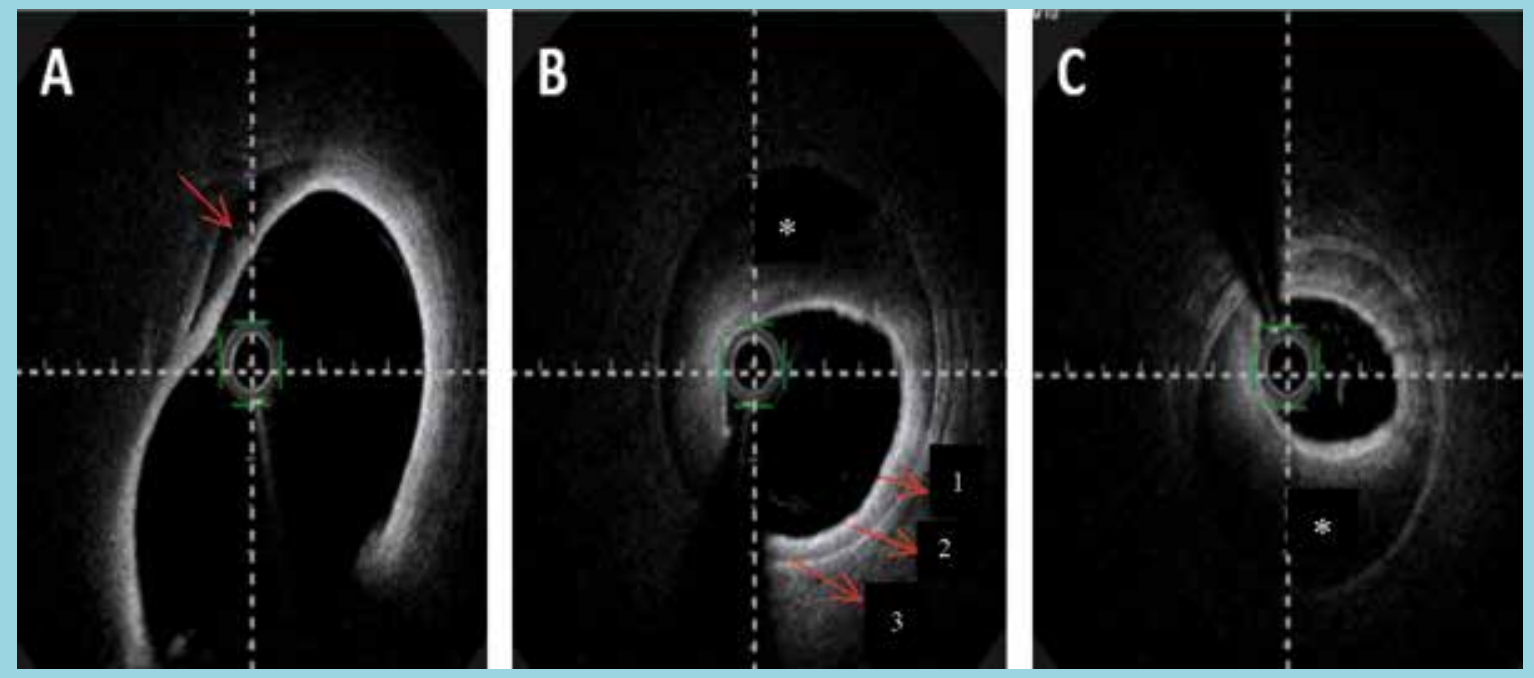
luego de 8 días, en tratamiento con aspirina, clopidogrel, atorvastatina y atenolol.

Dos semanas post alta, presentó episodios recurrentes y auto limitados de dolor retroesternal opresivo, en reposo, asociados a disnea y diaforesis, por lo cual fue hospitalizada nuevamente. A su ingreso, sin dolor torácico y estable del punto de vista clínico, con un electrocardiograma ilustrado en la Figura 4.

Se realizó una nueva coronariografía que mostró un estrechamiento del lumen de la descendente anterior, comparable al evidenciado en la coronariogafía previa, pero con un lumen residual aceptable y sin compromiso del flujo coronario (Figura 5A). Se decidió nuevamente manejo médico, con anticoagulación con heparina por 5 días. Luego de esto se realizó una nueva coronariografía de control (Figura 5B), que demostró una regresión del estrechamiento luminal de la descendente anterior. La paciente se mantuvo hospitalizada por 10 días, asintomática y fue dada de alta con tratamiento antiagregante dual y beta bloqueo. En un control clínico tardío, ha permanecido en buenas condiciones, sin nuevos episodios de dolor torácico.

\section{Discusión:}

La disección espontánea de arteria coronaria es una entidad clínica poco frecuente, de causa desconocida, que afecta típicamente a mujeres jóvenes sin factores de riesgo cardiovascular clásicos ${ }^{1}$. Los reportes iniciales de disección coronaria, se basaban en los estudios de autopsia de muerte súbita cardiaca ${ }^{1,2}$. Se define como una separación de la pared arterial coronaria entre la íntima y media, o entre la media y la adventicia, por una hemorragia intra-

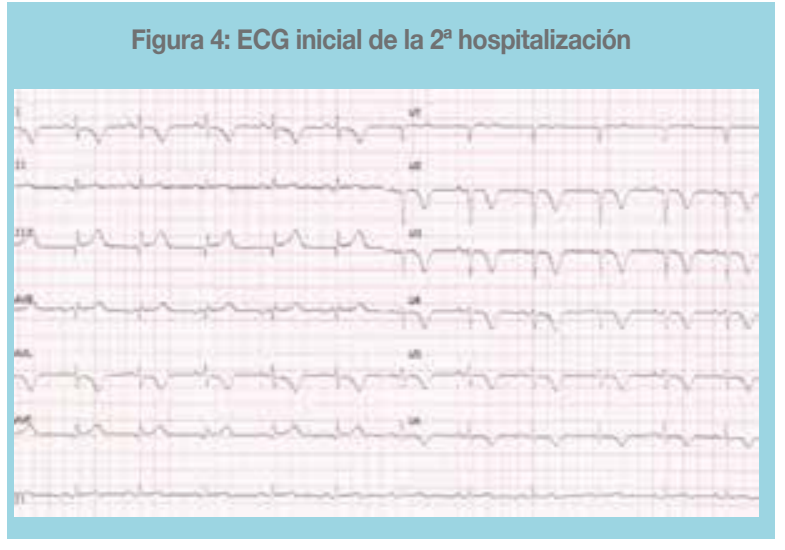

mural, creando así una falsa luz, con o sin un desgarro de la íntima. En los casos en que no hay desgarro intimal se le denomina Hematoma Intramural, el cual se cree sería causado por rotura de los vasa vasorum ${ }^{3}$. La extensión del falso lumen de la disección o del hematoma intramural puede comprimir la luz arterial verdadera, afectando el flujo anterógrado causando isquemia o infarto ${ }^{3}$.

Si bien clásicamente se ha implicado al embarazo, puerperio $^{3} \mathrm{o}$ a enfermedades del mesénquima, como entidades causales, actualmente la mayor asociación de la disección coronaria espontánea es con la displasia fibromuscular, la cual se ha observado hasta en el $72 \%$ de los pacientes ${ }^{4}$.

La Coronariografía permite el diagnóstico de la disección coronaria ${ }^{5}$ por la solución de continuidad de la íntima, elemento distintivo de esta enfermedad ${ }^{1}$. Por otra parte, el estrechamiento del lumen de la arteria coronaria causado por un hematoma intramural es un hallazgo más sutil, que se puede confundir con enfermedad aterosclerótica ${ }^{1} \mathrm{o}$

Figura 5: A: Se observa un estrechamiento del lumen de la descendente anterior, comparable al evidenciado en la coronariogafía previa, pero con un lumen residual aceptable y sin compromiso del flujo coronario; B: regresión del estrechamiento en la ADA.
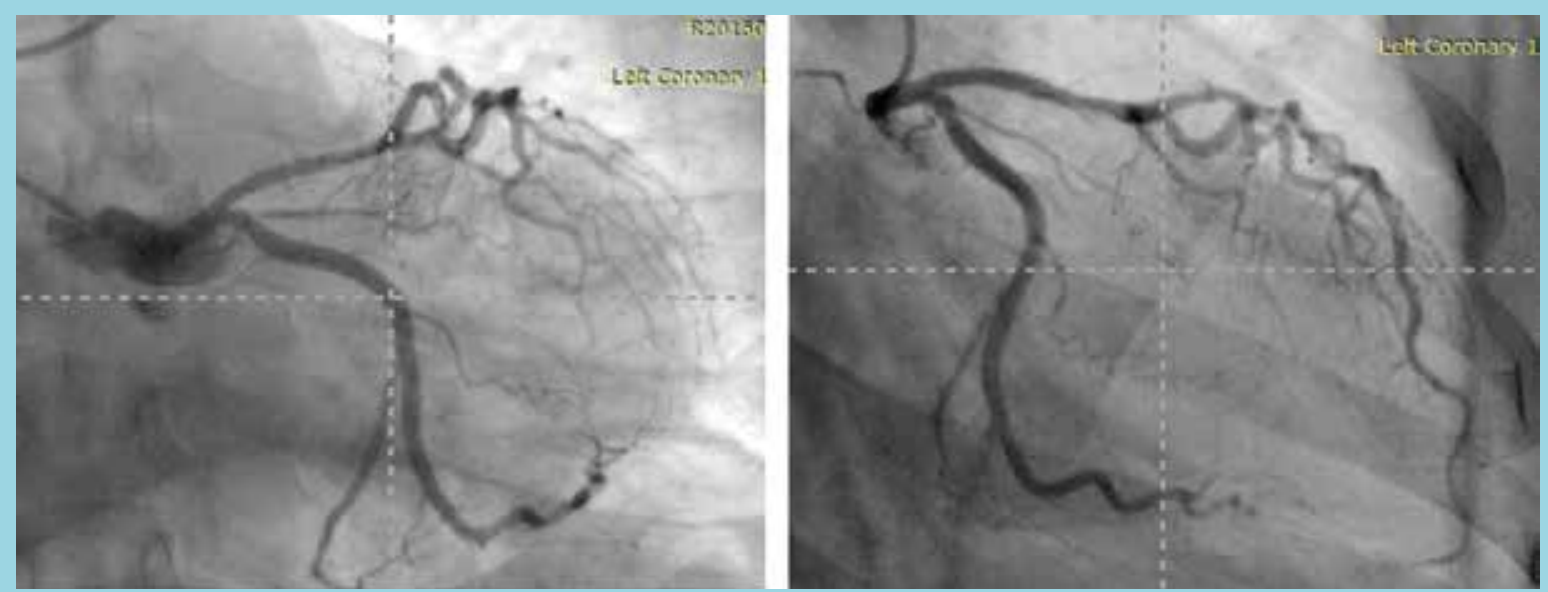
vaso espasmo coronario ${ }^{6}$. Por ello, se estima que la incidencia de esta entidad es subdiagnosticada ${ }^{1,4}$. Para superar las limitaciones de la angiografía coronaria, las técnicas de diagnóstico intravascular proporcionan imágenes de alta resolución de la pared del vaso. El ultrasonido intravascular (IVUS) (resolución 150 micras) y OCT (resolución 15 micras) son de utilidad para establecer el diagnóstico y guiar intervenciones terapéuticas ${ }^{1,5}$.

Este caso refleja una presentación dramática de un hematoma intramural, con paro cardíaco y evidencias de isquemia en la pared anterior. En un serie prospectiva de 168 pacientes con disección coronaria espontánea, el $26 \%$ de los pacientes se presentó como infarto con supradesnivel del segmento ST y el $4 \%$ presento Taquicardia Ventricular/ Fibrilación Ventricular. Tal como en el presente caso. la arteria descendente anterior fue la más frecuentemente comprometida ( $41 \%)$

En la actualidad existe consenso de que el tratamiento médico debiese ser la estrategia inicial en estos pacientes, toda vez que no exista compromiso del flujo coronario o isquemia miocárdica refractaria. De hecho, en la mayoría de los pacientes el tratamiento conservador se asocia a buenos resultados, demostrándose muchas veces curación espontánea de la disección ${ }^{4,5}$. Sin embargo, el manejo médico óptimo no se encuentra aclarado. Se ha recomendado aspirina y $\beta$-bloqueo a largo plazo. La terapia adicional antiplaquetaria con clopidogrel también se administra típicamente de 1 a 12 meses, aunque la evidencia que soporta su uso es escasa. El papel de la terapia anticoagulante para estos casos es controvertido ${ }^{3}$.

La terapia de revascularización se limita a aquellos pacientes con fracaso de la terapia médica. En la serie de Clínica Mayo ${ }^{6}$, la tasa de falla de procedimiento en pacientes sometidos a angioplastía coronaria fue de $53 \%$. En el subgrupo de pacientes que se presentó con flujo del vaso preservado, la tasa de fracaso de la angioplastía fue igualmente alta $(50 \%)$ y $13 \%$ requirió cirugía de revascularización de emergencia. En el grupo conservador, por el contrario, $90 \%$ tuvo un curso sin complicaciones en el hospital y solo $10 \%$ experimentó progresión de la disección temprana que requirió revascularización.

Este caso ejemplifica la utilidad de técnicas de imagen intravascular, como el OCT, para el diagnóstico de entidades complejas y poco frecuentes en patología coronaria.

\section{Referencias:}

1. ALFONSO F. Spontaneous coronary artery dissection: new insights from the tip of the iceberg? Circulation 2012; 126:667-70.

2. BASSO C, MORGAGNI GL, THIENE G. Spontaneous coronary artery dissection: a neglected cause of acute myocardial ischaemia and sudden death. Heart 1996; 75:451-54.

3. SAW J. Spontaneous coronary artery dissection. Can J Cardiol 2013; 29:1027-33.

4. SAW J, AYMONG E, SEDLAK T, BULLER CE, STAROVOYTOV A, RICCI D, et al. Spontaneous coronary artery dissection: association with predisposing arteriopathies and precipitating stressors and cardiovascular outcomes. Circ Cardiovasc Interv 2014; 7:645-55.

5. ALFONSO F, PAULO M, LENNIE V, DUTARY J, BERNARDO E, JIMÉNEZ-QUEVEDO P, et al. Spontaneous coronary artery dissection: long-term follow-up of a large series of patients prospectively managed with a "conservative" therapeutic strategy. JACC Cardiovasc Interv 2012; 5:1062-70.

6. TWEET MS, ELEID MF, BEST PJ, LENNON RJ, LERMAN A, RIHAL CS, et al. Spontaneous coronary artery dissection: revascularization versus conservative therapy. Circ Cardiovasc Interv 2014; 7:777-86 


\title{
Calidad de las Tromboplastinas utilizadas en el Laboratorio Clínico y en los equipos POCT y su impacto en la dosificación de acenocumarol en pacientes con terapia anticoagulante oral
}

\author{
Elena Nieto ${ }^{1}$, Ángela Roco ${ }^{2}$, Marcelo Moreno ${ }^{3}$, Victoria Carrasco ${ }^{3}$, Angel Geraldo $^{3}$, Betty \\ Rodríguez ${ }^{4}$, Omar Zagmutt ${ }^{5}$, Carlos Gallardo ${ }^{6}$, Jacqueline Peters ${ }^{7}$. \\ 1. Médico especialidad Laboratorio Clínico, Policlínico de Tratamiento Anticoagulante CDT-Hospital San Juan de Dios. \\ 2. Bioquímico, Coordinación de Unidades de Apoyo, Departamento Coordinación de la Red Asistencial, Servicio de \\ Salud Metropolitano Occidente. \\ 3. Tecnólogo Médico, Laboratorio Clínico Hospital San Juan de Dios. \\ 4. Químico Farmacéutico, Laboratorio Clínico Hospital San Juan de Dios. \\ 5. Médico, Departamento de Procesos Asistenciales, Servicio de Salud Metropolitano Occidente. \\ 6. Médico, Departamento Coordinación de la Red Asistencial, Servicio de Salud Metropolitano Occidente. \\ 7. Bioquímico, Jefe Laboratorio Clínico Hospital San Juan de Dios.
}

Antecedentes: El monitoreo del tratamiento con anticoagulantes cumarínicos se realiza a través del INR (International Normalized Ratio) que es el parámetro estandarizado del Tiempo de Protrombina. Las recomendaciones de la OMS indican que la precisión en el cálculo del INR puede ser mejorada usando reactivo de tromboplastina con Índice de Sensibilidad Internacional (ISI) bajo, considerándose como ISI de referencia internacional el valor 1,0. Debido a incongruencias observadas en los INR de pacientes controlados en el Servicio de Salud Metropolitano Occidente, comparando valores de muestra venosa con resultados de INR capilar obtenidos en el mismo paciente el mismo día y hora (con reactivos Tromboplastina de distinto ISI), se efectuó un ensayo clínico cruzado entre los distintos métodos.
Materiales y métodos: En 100 pacientes se comparó INR venoso con dos tromboplastinas de diferente ISI $(1,3$ y 1,0$)$ vs aquel efectuado con muestra capilar (ISI 1,0).

Resultados: Los resultados del estudio muestran que a partir de valores de INR 3,0 las determinaciones obtenidas usando Tromboplastina de cerebro de conejo ISI $=1,3$ subestiman el valor de INR para un mismo paciente y una misma muestra.

Conclusiones: El uso de Tromboplastina recombinante humana ISI 1,0 permite evitar la subestimación del INR en pacientes con mayor riesgo tromboembólico (indicación de INR objetivo más alto). Por ello, este método se adoptó en el control del TACO en pacientes controlados en el Servicio de Salud Occidente. 